\title{
Towards an Implantable Vestibular Prosthesis: The Surgical Challenges
}

\author{
Timothy G. Constandinou* ${ }^{* \dagger}$, Julius Georgiou*, Charalambos C. Doumanidis ${ }^{\ddagger}$, and Chris Toumazou ${ }^{\dagger}$ \\ ${ }^{*}$ Holistic Electronics Research Lab, Dept. of Electrical \& Computer Engineering, University of Cyprus, Nicosia 1678, Cyprus \\ ${ }^{\dagger}$ Institute of Biomedical Engineering, Imperial College of Science, Technology and Medicine, London SW7 2AZ, UK \\ ${ }^{\ddagger}$ Department of Mechanical \& Manufacturing Engineering, University of Cyprus, Nicosia 1678, Cyprus \\ Email: t.constandinou@\{ucy.ac.cy,imperial.ac.uk\}, \{julio,cdoumani\}@ucy.ac.cy, c.toumazou@imperial.ac.uk
}

\begin{abstract}
Restoring the sensation of inertia in individuals with balance-related impairments is achievable through development of an artificial vestibular prosthesis, based on the cochlear implant paradigm. The vestibular system, however, involves added complexity in surgical methods required for reliable implantation, thus limiting widespread feasibility. This work aims to support research in developing surgical technology for precisely accessing inside the vestibular organ. Issues relating to surgical procedure, microrobotics and medical imaging are discussed, with the goal being the precise and safe implantation of microelectrode arrays. This paper presents the challenges modern surgical technology would encounter in adopting such a procedure and proposes possible solutions, aiming to promote and motivate research in this direction.

Index Terms-Vestibular, Prosthesis, Implantable, Microsurgery, Medical Imaging
\end{abstract}

\section{INTRODUCTION}

Neuroprosthetics or neural engineering is a relatively new topic but it has already shown its potential. Since the application of this science, it has already significantly improved the quality of life of over 60,000 individuals who previously suffered from severely impaired hearing or total deafness. Today, through use of cochlear implants, children born totally deaf can enjoy going to regular schools and communicating normally.

Handicapped individuals suffering from dizziness and balance disorders can also benefit from the progress made in cochlear prosthetics, combined with modern microelectromechanical systems (MEMS). The inner ear's vestibular system provides cues about self-motion and help stabilise vision during movement. Damage to this system can result in dizziness, imbalance, blurred vision and instability in locomotion, a leading cause of death in the elderly. Restoration of balance can therefore be achieved by bypassing a dysfunctional element in the vestibular pathway using artificial stimulation. There are a number of sites along the vestibular pathway that can be tapped into; the ampullae, the scarpa's ganglion and individual nerve bundles.

A single channel artificial semicircular canal has been developed by Gong and Merfeld [1], demonstrating positive improvement in vestibulo-ocular response (VOR), in addition to long-term adaptability to artificial electrical stimulation in guinea pigs with surgically plugged semicircular canals [2]. Shkel et al. [3] developed a unilateral prosthesis, incorporating a custom MEMS-based inertia sensor, aimed for the squirrel monkey model. Santina et al. [4] proceeded to develop a three-axis device, used to stimulate all three ampullary nerveendings in Chinchillas. They reported that 3-D oculography measured significant responses in perpendicular planes to those which had been artificially stimulated. This was confirmed by micro-CT (computed tomography) imaging, revealing that surgical positioning of a single microelectrode was in close proximity of all three ampullar targets.

Our work intends to develop an miniature, low power, MEMSbased vestibular implant chip, sensing all six degrees of freedom (i.e. 3-axis linear and 3-axis radial acceleration). This additionally requires to emulate the otolith endorgan functionality, effectively generating a planar stimulus, targeting the afferent (directionally sensitive) fibres. However, based on the findings of [4], it is anticipated that such a prosthesis (i.e. providing multi-channel stimulus) would demand advanced and precise microsurgery. Fig. 1 illustrates the mammalian vestibular system to scale and thus highlights why such precision is needed. This paper therefore aims to address these issues and motivate related research within parallel disciplines including: medical robotics, medical imaging and surgical technology.

Section I has begun by introducing the field of neuroprosthetics and more specifically relating to an implantable vestibular prosthesis. Expected challenges and technical constraints in the surgical procedure are then outlined in section II. Sections III and IV continue by reviewing current state-of-the-art medical imaging and microrobotic technologies applicable to this field, identifying strengths and weaknesses of each approach in the context of the target application. Following in section $\mathrm{V}$ is a proposal of an image-guided robotic platform for vestibular implant surgery, describing how it would overcome limitations of other techniques. Finally, section VI ends by summarising the key points mentioned in this paper.

\section{Vestibular IMPlant SURGery}

The concept behind this implantable prosthesis is to restore vestibular balance cues by overriding the fluid-mechanic transduction within the semicircular canals and otolith organs, using artificial apparatus interfacing to the vestibulocochlear (VIII) nerve. Multiaxis inertia sensors, mounted externally to the inner ear, are used to feed processed (electrical) stimuli to the nerve endings of the vestibular branch of the VIII'th nerve. This terminates as the afferent

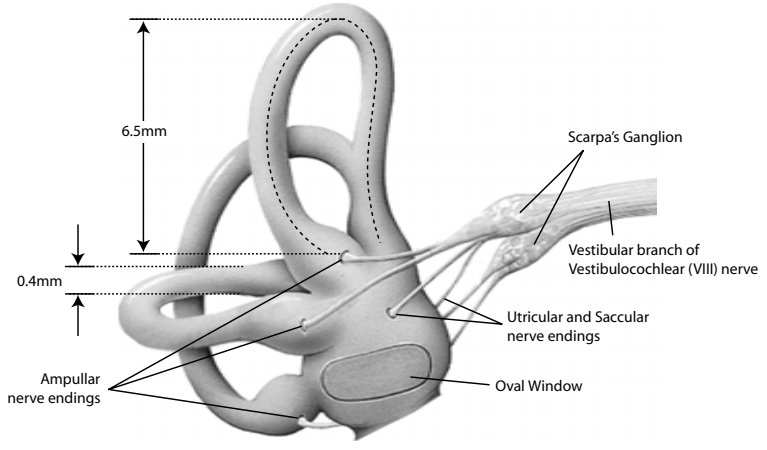

Fig. 1. Diagram of the mammalian vestibular system including the various nerve bundle endings, i.e. the target stimulation sites. This illustrates the minute nature of this organ and close proximity between different sensing apparatus (adjacent stimulation channels). 
and efferent fibres of the hair-cells within the ampullae (semicircular canals) and maculae (otolith organs).

The surgical procedure therefore involves precise placement of micro-electrode pairs to be in close proximity with the hair cell membranes, such that artificial electrical stimulation can efficiently modulate Scarpa's Ganglion to generate action potentials.

The intricate anatomy and topology of the vestibular system demands high-resolution medical imaging technology to locate and identify the specific features. Furthermore, as the vestibular apparatus are completely encapsulated within the temporal bone, a penetrative imaging modality is required. To perform microsurgery to such constraints a high-resolution image-guided procedure needs to be adopted, controlling microrobotic surgical tools. Real-time imaging is required to ensure reliable and accurate coupling between the surgical reference (tool) and target (site). The surgical task would be to machine precise cavities (i.e. with sub- $200 \mu \mathrm{m}$ resolution) within the bone for micro-electrode insertion.

Such precision is required for two reasons: (i) to ensure that the machined micro-cavities do not fully penetrate the bone, i.e. pierce into the membranous labyrinth, thus causing a leak of endolymph. This would result in the drying up of the inner ear, leading to loss of hearing and balance in that ear. (ii) So the micro-electrodes can be positioned to as close proximity as possible to the stimulation sites, for artificial stimulation to be efficient and microsurgery to be of minimal risk. Additionally, precise positioning is required such that any cross-talk (with adjacent stimulation sites) or interference is minimised.

\section{MedicAl Imaging}

There are currently several methods of non-invasive imaging for clinical use. Three such technologies are: computed tomography (CT) scan, magnetic resonance imaging (MRI) and ultrasound (US) imaging. These are compared in Table III.

High Resolution Computed Tomography (HRCT) scanning excels in the evaluation of bone and air space anatomy and disorders of the temporal bone. Using recently developed software reconstruction techniques, pixel size can be resolved down to below $250 \mu \mathrm{m}$. On the other hand, High Definition Magnetic Resonance Imaging (HDMRI) is very helpful in the imaging of the membranous labyrinth, facial and vestibulocochlear nerves [8]. However, insensitivity to bone and calcified structures are disadvantages to its use [9]. A third alternative is ultrasound (or ultrasonography), recently evolving to display realtime 3D images [10], with commercially available systems having a minimum pixel size of down to $200 \mu \mathrm{m}$. Moreover, specific high resolution ultrasonography has been developed for imaging of the inner ear [7], with the ability of achieving a minimum pixel size of below $100 \mu \mathrm{m}$.

The limitation of both HRCT and HDMRI is that of portability and non-applicability for real-time imaging. MRI and CT scanners are conventionally large, stand-alone imaging apparatus and thus cannot be used to provide real-time imaging of live surgery. On the other hand, ultrasonography is portable and therefore suitable for real-time imaging, however it has limited usefulness due to its inability to penetrate (i.e. image beyond) bone and/or gaseous voids.

A solution being increasingly used in cardiovascular- [11] and neuro- [12] surgery is to employ an image registration technique to combine two imaging techniques, thus to benefit from both methods. Typically, pre-operative images are obtained using HDMRI or HRCT and registered onto live US images. One method of achieving this is by defining a number of fiducial (i.e. reference) points on both reference and target and applying a morphing algorithm such that the high resolution pre-operative image is mapped onto the real-time US image, thus creating a real-time high definition image suitable for guiding microrobotic surgery.

We believe this technique could be directly applicable to vestibular implant surgery, additionally combining both HDMRI and HRCT imaging to form a hybrid reference image with both excellent soft tissue and bony detail. Similar techniques have recently been used to simultaneously image pituitary and anterior skull base [13]. In our case, this would be used in image guided surgery to provide detail on both the temporal bone anatomy and inner membranous labyrinth structures.

\section{ROBOTIC SURGERY}

Robot-guided or Robotic-assisted surgery has recently emerged; providing surgeons with motor-actuated surgical tools, increasing precision, advancing miniaturisation and reducing risk of infection. Currently, the system in most widespread use is the Da Vinci robot (Intuitive Surgical, US), the first such system with full FDAapproval for clinical use. This is aimed towards generic minimally invasive surgery, mimicking a surgeons hand movement at a $3 \mathrm{D}$ visualisation and control console with stabilised robot-assisted motion at the surgical target. Other systems, designed to be application specific include the RoboDoc (Integrated Surgical Systems, CA) for orthopaedic surgery, and the Pathfinder (Prosurgics, UK) and Neuromate (Integrated Surgical Systems, CA), for neurosurgery. These are all based upon an image-guided computer-controlled robotic arm with target stabilisation and/or tracking. Although it would seem that a similar tool could be adopted for vestibular implant surgery, in fact, such systems cannot achieve the required accuracy. For instance, studies have measured the application accuracy of the Pathfinder system to be on average $2.7 \mathrm{~mm}$ [14], compared to $1.95 \pm 0.44 \mathrm{~mm}$ for the Neuromate [15]. In the case of the Neuromate, it is noted that employing a frame-based (ZD) mounting configuration can further improve accuracy, achieving $0.86 \pm 0.32 \mathrm{~mm}$. The relatively low precision of such systems is attributed to the drive mechanism of the robotic arm, often pulley/belt driven and therefore having limited open-loop accuracy. Furthermore, autonomous robotic systems typically employ real-time tracking for patient/robot registration. Such techniques often use ultrasonic [15] or infra-red [16] transducers for alignment, using bone-screwed references. However, such techniques, in general achieve at best $1 \mathrm{~mm}$ registration accuracy.

An alternative approach to using a floor (or bed) mounted robotic arm, is to use a bone-mounted miniature robot. The SpineAssist (Mazor Surgical Technologies, US) system, initially developed for screw insertion into spinal fusion has recently been adopted for neurosurgery (MARS robot) [17]. Through miniaturisation, it is expected to improve accuracy, firstly due to decreased dimensions, thus using actuator mechanisms of higher resolution and secondly, due to the possibility of mounting the entire system to the target reference, eg. mounting to bone by alignment pins or screws.

In general, the target localisation error of any robot-guided or assisted surgical tool can be attributed to the following four factors: (i) the repeatability of the robot, (ii) the resolution (accuracy) of the reference image, (iii) the registration between the reference image and target and (iv) deformation/movement of the target. By examining these factors individually, it can be established how to achieve substantial improvement in application accuracy.

\section{Vestibular Implant Robotic Microsurgery}

This section proposes a miniature bone-mounted 5-axis robot for temporal bone micro-machining. 
TABLE I

COMPARATIVE REVIEW OF HIGH RESOLUTION IMAGING TECHNOLOGIES SUITABLE FOR VISUALISATION OF THE VESTIBULAR APPARATUS

\begin{tabular}{|c|c|c|c|c|}
\hline Method & Parameter Measured & Medical Applications & Min. Pixel Size & Equipment Manufacturers \\
\hline $\begin{array}{l}\text { Transmission and } \\
\text { emission computed } \\
\text { tomography (CT) [5] }\end{array}$ & Density and average atomic number & $\begin{array}{l}\text { Anatomy, mineral content, flow and } \\
\text { permeability from movement of } \\
\text { contrast material }\end{array}$ & $100-250 \mu \mathrm{m}$ & $\begin{array}{l}\text { GE Healthcare, Siemens, } \\
\text { Philips, Toshiba, Hitachi }\end{array}$ \\
\hline $\begin{array}{l}\text { Magnetic resonance } \\
\text { imaging (MRI) [6] }\end{array}$ & $\begin{array}{l}\text { Concentrations, relaxation parameters } \\
\mathrm{T} 1 \text {, and } \mathrm{T} 2 \text { and frequency shifts due } \\
\text { to chemical form }\end{array}$ & Anatomy, edema, flow & $250-500 \mu \mathrm{m}$ & $\begin{array}{l}\text { Fonar, Philips, Siemens, } \\
\text { GE Healthcare, Hitachi, } \\
\text { Toshiba }\end{array}$ \\
\hline Ultrasound (US) [7] & $\begin{array}{l}\text { Acoustic impedance mismatches, sound } \\
\text { velocity, attenuation, frequency } \\
\text { shifts due to motion }\end{array}$ & $\begin{array}{l}\text { Anatomy, tissue structure } \\
\text { characteristics, flow }\end{array}$ & $200-500 \mu \mathrm{m}$ & $\begin{array}{l}\text { Hewlett Packard, Siemens, } \\
\text { Toshiba, Hitachi, Kontron, } \\
\text { Shimadzu, Fukuda Denshi }\end{array}$ \\
\hline
\end{tabular}

\section{A. Micro-machining Robot}

The system consists of a 5 -axis robot consisting of a $\mathrm{X} / \mathrm{Y}$-axis bed positioning system [18] with $\mathrm{Z} / \theta_{X} / \theta_{Y}$-axis positioning at the tool head for depth and entry angle control. This is based on the configuration often used in flatbed Computer Numerical Control (CNC) technology for sheet machining (milling); in which it is common to achieve sub-10 $\mu m$ resolution. This high accuracy is attributed to lead- or ball-screw based actuators for positioning [19]. As in [17], the actuated platform will hold a drill guide to position the high speed surgical spindle required for the temporal bone (mastoid) micro-machining. The possibility of using a surgical laser for bone removal has been ruled because of the risk in hair cell nerve damage due to heat shock.

\section{B. Patient/Robot Registration}

To overcome patient/robot registration errors mentioned previously, our approach chooses to use a bone-mounted miniature robot to ensure both patient and robot are rigidly coupled. The technique to be used, as in [17], requires a two part frame, consisting of: (i) the reference that is bolted onto the target (i.e. the patients temporal bone), and (ii) the microrobotic frame. These two components must be designed such that they can be easily separated but assemble together rigidly. Additionally, a head clamp can be used to relieve strain on the mounting screws, i.e. due to weight of robot.

\section{C. $X / Y / Z$ Calibration}

Precise calibration and registration of microrobotic head-position is achievable using a CCD/CMOS camera with optical magnification, mounted a pre-calibrated displacement from the drill guide mount. Typically, two alignment marks (fiducial points) made on the target, i.e. the temporal bone, can be used to form an precise reference, both optically by the robot (through the camera) and within the reference image, i.e. HDMRI/HRCT slices, thus providing an excellent alignment method. Furthermore, the camera can be used in conjunction with pre-defined tool bits (eg. $90^{\circ}$ tip engraving tool) to visualise/measure the depth, thus providing a method for accurately aligning the Z-axis.

\section{Proposed Surgical Procedure}

For the precise insertion of microelectrodes within the temporal bone for the vestibular prosthesis, we propose the following sequence to facilitate the robotic microsurgery:

1) Pre-operative screw-insertion planning: HRCT slices are taken to locate vicinity of labyrinth within temporal bone and to define the position of frame-mount screws and fiducial markers.
2) Screw mounting: Initial surgical procedure to insert mount screws into the temporal bone. Reference frame is fastened rigidly onto the temporal bone.

3) Frame mounting: Microrobotic drill guide attached to reference frame, head clamp fastened (to relieve strain on mounting screws) and high speed spindle loaded.

4) Fiducial marking: Shallow holes are engraved onto temporal bone using $1 \mathrm{~mm}$ drill tool.

5) Frame removal: Head clamp, drill guide and spindle removed from patient (reference frame remains mounted).

6) Pre-operative surgical path planning: New HDMRI/HRCT slices taken to define target microelectrode positions and plan the surgical path, with reference to the engraved fiducial markers (these will be visible on the HDMRI/HRCT images).

7) Frame mounting: Microrobotic drill guide again attached to reference frame, head clamp fastened (to relieve strain on mounting screws) and high speed spindle loaded.

8) $X / Y$ registration: Bone surface scanned using microrobotic head-mounted optical camera to locate fiducial points, i.e. X/Yreference.

9) Z registration: Near fiducial points, temporal bone surface profiled semi-automatically using a $45^{\circ}$ tipped engraving tool to mark the surface. Subsequently, by measuring the groove width (using camera), the depth can be precisely determined (to within $\pm 10 \mu m$ ).

10) Microsurgery: The robotic microsurgery can then be initiated; to drill holes into the temporal bone for microelectrode insertion.

Using the above described technology and methodology, the machined cavities will be aligned to the HDMRI/HRCT data with sub-100 $\mu \mathrm{m}$ alignment precision. This is achievable as all methods mentioned are commonly used in CNC-based printed circuit board prototyping technology, in which a better than $10 \mu \mathrm{m}$ registration precision is achievable over an A3-size bed.

\section{CONCLUSION}

This paper has identified a specific application in which advances in parallel research fields could vastly benefit clinical feasibility. Based on the proven success of the cochlear implant, a vestibular prosthesis could have similar impact on affected individuals. Fundamentals of hair-cell neural transduction are identical for both cochlear and vestibular end-organs, both extending to form the vestibulocochlear (VIII) nerve. Vestibular implant technology is being developed by ourselves and at least three other groups around the world. We identify the field that could delay clinical application of vestibular 
implants is surgical implantation technology for precise, robust insertion of microelectrodes. Additionally, this would aid advances in apparatus for the recording of vestibular neurophysiology, at present being non-existent. For microelectrode implantation we propose a solution describing a bone-mounted miniature 5-axis robot for temporal bone micro-machining. We've addressed issues relating to image registration, surgical positioning precision and patient stabilisation. We aim for this discussion to spark off interest the relevant research communities, promoting much needed development in this vastly underdeveloped field.

\section{ACKNOWLEDGMENT}

This work was supported by the Cyprus Research Promotion Foundation (RPF), grant no.: $\Pi \Delta E-0505 / 07$. The authors would like to thank ENT Surgeon Neil Tolley for clinical insight and Dan Elson/George Mylonas for useful discussion on medical imaging and robotic technologies.

\section{REFERENCES}

[1] W. Gong and D. M. Merfeld, "System Design and Performance of a Unilateral Horizontal Semicircular Canal Prosthesis," IEEE Transactions on Biomedical Engineering, vol. 49, no. 2, pp. 175-181, 2002.

[2] R. F. Lewis, W. Gong, M. Ramsey, et al., "Vestibular adaptation studied with a prosthetic semicircular canal," Journal of Vestibular Research, vol. 12, pp. 87-94, 2003.

[3] A. M. Shkel, and F-G. Zeng, "An Electronic Prosthesis Mimicking the Dynamic Vestibular Function," Audiology and Neurotology, vol. 11, pp. 113-122, 2006.

[4] C. C. D. Santina, A. A. Migliaccio and A. H. Patel, "Electrical Stimulation to Restore Vestibular Function - Development of a 3-D Vestibular Prosthesis," Proceedings of the IEEE Conference on Engineering in Medicine and Biology Society, pp. 7380-7385, 2005.

[5] H. Lopponen, T. Holma, M. Sorri, et al., "Computed tomography data based rapid prototyping model of the temporal bone before cochlear implant surgery," Acta Otolaryngol Suppl., vol. 529, pp. 47-49, 1997.

[6] P. Hans, A. J. Grant, R. D. Laitt, et al., "Comparison of 3D Visualization techniques for depicting the scala vestibuli and scala tympani of the cochlea by using high-resolution MR imaging," Am. Journ. Neuroradiology, vol. 20, no. 7, pp. 1197-1206, 1999.

[7] D. L. Rothbaum, J. Roy, T. Mustufa, et al., "High-Resolution Ultrasonography of the Cochlea," Otology \& Neurotology, 2004.

[8] B. Arnold, L. Jager and G. Grevers, "Visualization of Inner Ear Structures by Three-Dimensional High-Resolution Magnetic Resonance Imaging," American Journal of Otology, vol. 17, pp. 480-485, 1996.

[9] D. W. Chakeres, M. Oehler, et al., Head and Neck Imaging, ch. Temporal bone imaging, pp. 1319-1350. Mosby, 3rd ed., 1996.

[10] A. Fenster, D. B. Downey and H. N. Cardinal, "Three-dimensional ultrasound imaging," Physics in Medicine and Biology, vol. 46, no. 5, pp. 67-99, 2001

[11] T. J. Makela, P. Clarysse, O. Sipila, et al., "A review of cardiac image registration methods," IEEE Transactions in Medical Imaging, vol. 21, no. 9, pp. 1011-1021, 2002.

[12] D. G. Gobbi, R. M. Comeau, B. K. H. Lee, et al., "Integration of intra-operative 3D ultrasound with pre-operative MRI for neurosurgical guidance," Proc. Int. Conf. IEEE Engineering in Medicine and Biology Society, vol. 3, pp. 1738-1740, 2000.

[13] J. L. Leong, P. S. Batra and M. J. Citardi, "CT-MR image fusion for the management of skull base lesions," Otolaryngology - Head and Neck Surgery, vol. 134, no. 5, pp. 868-876, 2006.

[14] P. S. Morgan, T. Carter, S. Davis, et al., "The application accuracy of the Pathfinder neurosurgical robot," Proc. Int. Conf. Computer Assisted Radiology and Surgery, vol. 1256, no. 3, pp. 561-567, 2003.

[15] Q. H. Li, L. Zamorano L, A. Pandya, et al., "The application accuracy of the NeuroMate robot-A quantitative comparison with frameless and frame-based surgical localization systems," Computer Aided Surgery, vol. 7 , no. 2 , pp. 90-98, 2002.

[16] R. M. Calvin, J. M. Fitzpatrick, M. Y. Yang, et al., "Registration of Head Volume Images Using Implantable Fiducial Markers," IEEE Trans. Medical Imaging, vol. 16, no. 4, pp. 447-462, 1997.
[17] M. Shoham, M. Burman, E. Zehavi, et al., "Bone-mounted miniature robot for surgical procedures: Concept and clinical applications," IEEE Trans. Robotics and Automation, vol. 19, no. 5, pp. 893-901, 2003.

[18] H. L. H. R. W. Burnette and R. V. Lukes, "Us patent 3,638,933: Precision x-y positioning table," 1970

[19] F. R. Chaffin, "Us patent 3,975,968: Precision lead screw assembly and nut," 1976 